International Mathematical Forum, 2, 2007, no. 51, 2543 - 2549

\title{
Common Fixed Point Theorems in Sequentially Compact Fuzzy Metric Spaces
}

\author{
K. P. R. Rao, K. R. K. Rao and T. Ranga Rao \\ Dept. of Applied Mathematics \\ Acharya Nagarjuna University-Nuzvid Campus \\ Nuzvid-521 201, A.P., India \\ kprrao2004@yahoo.com
}

\begin{abstract}
In this paper, we give common fixed point theorems in sequentially compact fuzzy metric space $(X, M, *)$ for pairs of weakly compatible mappings and for sequences of self mappings.
\end{abstract}

Mathematics Subject Classification: 47H10; 54H25

Keywords: Fuzzy metric space, weakly compatible pair, Common fixed points, sequentially compact

\section{Introduction and Preliminaries}

The concept of fuzzy sets was introduced initially by Zadeh [4] in 1965. George and Veeramani [1] modified the concept of fuzzy metric space introduced by Kramosil and Michalek [2]. In this paper we prove common fixed point theorems for pairs of weakly compatible self maps in a sequentially compact fuzzy metric space and also obtain two corollaries.

Definition 1.1 A binary operation $*:[0,1] \times[0,1] \longrightarrow[0,1]$ is a continuous t-norm if it satisfies the following conditions

1. * is associative and commutative,

2. * is continuous,

3. $a * 1=a$ for all $a \in[0,1]$,

4. $a * b \leq c * d$ whenever $a \leq c$ and $b \leq d$, for each $a, b, c, d \in[0,1]$.

Two typical examples of continuous t-norm are $a * b=a b$ and $a * b=$ $\min \{a, b\}$. 
Definition 1.2 A 3-tuple $(X, M, *)$ is called a fuzzy metric space if $X$ is an arbitrary (non-empty) set, $*$ is a continuous $t$-norm, and $M$ is a fuzzy set on $X^{2} \times(0, \infty)$ satisfying the following conditions for each $x, y, z \in X$ and $t, s>0$,

$$
\begin{aligned}
& \text { 1. } M(x, y, t)>0 \\
& \text { 2. } M(x, y, t)=1 \text { if and only if } x=y, \\
& \text { 3. } M(x, y, t)=M(y, x, t), \\
& \text { 4. } M(x, y, t) * M(y, z, s) \leq M(x, z, t+s), \\
& \text { 5. } M(x, y, .):(0, \infty) \longrightarrow[0,1] \text { is continuous. }
\end{aligned}
$$

Let $(X, M, *)$ be a fuzzy metric space. For $t>0$, the open ball $B(x, r, t)$ with center $x \in X$ and radius $0<r<1$ is defined by

$$
B(x, r, t)=\{y \in X: M(x, y, t)>1-r\} .
$$

Let $(X, M, *)$ be a fuzzy metric space. Let $\tau$ be the set of all $A \subset X$ with $x \in A$ if and only if there exist $t>0$ and $0<r<1$ such that $B(x, r, t) \subset A$. Then $\tau$ is a topology on $X$ induced by the fuzzy metric $M$. A sequence $\left\{x_{n}\right\}$ in $X$ converges to $x$ if and only if $M\left(x_{n}, x, t\right) \rightarrow 1$ as $n \rightarrow \infty$, for each $t>0$. A subset $A$ of $X$ is said to be F-bounded if there exists $t>0$ and $0<r<1$ such that $M(x, y, t)>1-r$ for all $x, y \in A$.

Lemma $1.3([5])$. Let $(X, M, *)$ be a fuzzy metric space. Then $M(x, y, t)$ is non-decreasing with respect to $t$, for all $x, y$ in $X$.

Lemma 1.4 (Proposition 1 of $[3])$. Let $(X, M, *)$ be a fuzzy metric space. Then $M$ is continuous function on $X^{2} \times(0, \infty)$.

Definition 1.5 Let $A$ and $S$ be mappings from a fuzzy metric space $(X, M, *)$ into itself. Then the mappings are said to be weakly compatible if they commute at their coincidence point, that is, $A x=S x$ implies that $A S x=S A x$.

Now we give

Definition $1.6(X, M, *)$ is said to be sequentially compact fuzzy metric space if every sequence in $X$ has a convergent sub sequence in it.

Let $\Phi$ be the set of all functions $\phi:[0,1]^{5} \longrightarrow[0,1]$ such that

(i) $\phi$ is non decreasing in all coordinates,

(ii) $\phi\left(t_{1}, t_{2}, t_{3}, t_{4}, t_{5}\right)$ is continuous in $t_{4}$ and $t_{5}$ and

(iii) $\phi(t, t, t, t, t)>t$ for every $t \in[0,1)$. 


\section{Main Results}

Hereafterwards,assume that $(X, M, *)$ be a sequentially compact fuzzy metric space with $t * t \geq t \forall t \in[0,1]$.

Theorem 2.1 Let $f, g$, Sand $T$ be self-mappings on $(X, M, *)$ such that

(1) $f(X) \subseteq T(X)$ and $g(X) \subseteq S(X)$,

$$
\begin{array}{rl}
{[1+a M(S x, T y, t)] *} & M(f x, g y, t) \\
\geq b[M(S x, T y, t) * M(f x, S x, t) * M(g y, T y, t) * \\
M(f x, T y, \alpha t) * M(g y, S x,(2-\alpha) t)]+ \\
\phi\left(\begin{array}{l}
M(S x, T y, t), M(f x, S x, t), M(g y, T y, t), \\
M(f x, T y, \alpha t), M(g y, S x,(2-\alpha) t)
\end{array}\right)
\end{array}
$$

for every $x, y \in X$, for all $t>0$ and for every $\alpha \in(0,2)$, where $\phi \in \Phi$ and $-1 \leq a \leq 0,0 \leq b \leq 1$,

(3) the pairs $(f, S)$ and $(g, T)$ are weakly compatible,

(4) $f$ and $S$ are continuous or $g$ and $T$ are continuous.

Then $f, g, S$ and $T$ have a unique common fixed point $p$ in $X$. Further $p$ is the unique common fixed point of $f$ and $S$ and of $g$ and $T$.

Proof. Suppose $f$ and $S$ are continuous.

For every $t>0$, let $m=\sup \{M(f x, S x, t): x \in X\}$.

Since $f$ and $S$ are continuous on sequentially compact fuzzy metric space,there exists $u \in X$ such that $m=M(f u, S u, t)$.

Since $f(X) \subseteq T(X)$, there exists $v \in X$ such that $f u=T v \ldots \ldots$ (5)

Suppose $m<1$.

Putting $x=u, y=v, \alpha=1-q_{1}, q_{1} \in(0,1)$ in (2) we have

$$
\begin{array}{rl}
{[1+a M(S u, T v, t)] *} & M(f u, g v, t) \\
\geq & b[M(S u, T v, t) * M(f u, S u, t) * M(g v, T v, t) * \\
& \left.M\left(f u, T v,\left(1-q_{1}\right) t\right) * M\left(g v, S u,\left(1+q_{1}\right) t\right)\right]+ \\
\phi\left(\begin{array}{l}
M(S u, T v, t), M(f u, S u, t), M(g v, T v, t), \\
M\left(f u, T v,\left(1-q_{1}\right) t\right), M\left(g v, S u,\left(1+q_{1}\right) t\right)
\end{array}\right) \\
{[1+a m] * M(T v, g v, t) \geq} & b\left[m * m * M(g v, T v, t) * 1 * m * M\left(g v, T v, q_{1} t\right)\right] \\
& +\phi\left(m, m, M(g v, T v, t), 1, m * M\left(g v, T v, q_{1} t\right)\right)
\end{array}
$$

Letting $q_{1} \rightarrow 1$, we have

$$
\begin{aligned}
{[1+a m] * M(T v, g v, t) \geq } & b[m * M(T v, g v, t)] \\
& +\phi(m, m, M(g v, T v, t), 1, M(g v, T v, t) * m)
\end{aligned}
$$




$$
M(T v, g v, t) \geq \phi(m, m, M(g v, T v, t), 1, M(g v, T v, t) * m)
$$

If $m \geq M(g v, T v, t)=r$ then using $t * t \geq t$ we have $r=M(g v, T v, t) \geq \phi(r, r, r, r, r)>r$. It is a contradiction. Hence we have $m<M(g v, T v, t)$

Since $g(X) \subseteq S(X)$, there exists $w \in X$ such that $g v=S w$.

Now $M(f w, S w, t) \leq m<1$. From (2), with $\alpha=1+q_{2}, q_{2} \in(0,1)$ we have

$$
\begin{array}{rl}
{[1+a M(S w, T v, t)] *} & M(f w, g v, t) \\
\geq & b[M(S w, T v, t) * M(f w, S w, t) * M(g v, T v, t) * \\
& \left.M\left(f w, T v,\left(1+q_{2}\right) t\right) * M\left(g v, S w,\left(1-q_{2}\right) t\right)\right]+ \\
\phi\left(\begin{array}{l}
M(S w, T v, t), M(f w, S w, t), M(g v, T v, t), \\
M\left(f w, T v,\left(1+q_{2}\right) t\right), M\left(g v, S w,\left(1-q_{2}\right) t\right)
\end{array}\right) \\
{[1+a M(g v, T v, t)] * M(f w, S w, t)} \\
\geq & b\left[\begin{array}{l}
M(g v, T v, t) * M(f w, S w, t) * M(g v, T v, t) * \\
\left.M(f w, S w, t) * M\left(g v, T v, q_{2} t\right) * 1\right]+
\end{array}\right. \\
\phi\left(\begin{array}{l}
M(g v, T v, t), M(f w, S w, t), M(g v, T v, t), \\
M(f w, S w, t) * M\left(g v, T v, q_{2} t\right), 1
\end{array}\right)
\end{array}
$$

Letting $q_{2} \rightarrow 1$, we have

$$
\begin{gathered}
{[1+a M(g v, T v, t)] * M(f w, S w, t)} \\
\geq b[M(g v, T v, t)] * M(f w, S w, t)+ \\
\phi\left(\begin{array}{c}
M(g v, T v, t), M(f w, S w, t), M(g v, T v, t) \\
M(g v, T v, t) * M(f w, S w, t), 1
\end{array}\right) \\
M(f w, S w, t) \geq \phi\left(\begin{array}{c}
M(g v, T v, t), M(f w, S w, t), M(g v, T v, t), \\
M(g v, T v, t) * M(f w, S w, t), 1
\end{array}\right) .
\end{gathered}
$$

If $M(g v, T v, t) \geq M(f w, S w, t)=s$ then

$$
M(f w, S w, t) \geq \phi(s, s, s, s, s)>s
$$

It is a contradiction. Hence we have $M(g v, T v, t)<M(f w, S w, t)$

Now from definition of $m$ and (8), (6) we have

$m \geq M(f w, S w, t)>M(g v, T v, t)>m$. It is a contradiction. Hence $m=1$.

Thus $f u=S u$. 
Suppose $M(g v, T v, t)<1$. Then from (2) with $\alpha=1$ we have

$$
\begin{array}{r}
{[1+a M(S u, T v, t)] * \begin{array}{r}
M(f u, g v, t) \\
\geq b[M(S u, T v, t) * M(f u, S u, t) * M(g v, T v, t) * \\
M(f u, T v, t) * M(g v, S u, t)]+ \\
\phi\left(\begin{array}{l}
M(S u, T v, t), M(f u, S u, t), M(g v, T v, t), \\
M(f u, T v, t), M(g v, S u, t)
\end{array}\right) \\
{[1+a 1] * M(T v, g v, t) \geq b[1 * 1 * M(g v, T v, t) * 1 * M(g v, T v, t)]+} \\
\phi(1,1, M(g v, T v, t), 1, M(g v, T v, t))
\end{array}} \\
M(T v, g v, t) \geq \phi(1,1, M(g v, T v, t), 1, M(g v, T v, t))>M(g v, T v, t) .
\end{array}
$$

It is a contradiction. Hence $g v=T v$..........

Thus $S u=f u=T v=g v=p$, say

Since the pair $(f, S)$ is weakly compatible we have

$f p=f f u=f S u=S f u=S S u=S p \ldots \ldots(12)$.

Suppose $r=M(p, f p, t)<1$. From (2) with $\alpha=1, x=p, y=v$ we have

$$
\begin{aligned}
& \begin{array}{l}
{[1+a M(S p, p, t)] * M(f p, p, t)} \\
\geq b[M(S p, p, t) * 1 * 1 * M(f p, p, t) * M(p, S p, t)]+ \\
\quad \phi(M(S p, p, t), 1,1, M(f p, p, t), M(p, S p, t)
\end{array} \\
& M(f p, p, t) \geq \phi(r, r, r, r, r)>r .
\end{aligned}
$$

It is a contradiction.

Hence $f p=p$. Thus $S p=f p=p$...(13)

Since the pair $(g, T)$ is weakly compatible we have $g p=T p$. Using (2) with $\alpha=1, x=u, y=p$ we can show that $g p=p$. Thus $T p=p=g p \ldots .(14)$.

Hence $p$ is a common fixed point of $f, g, S$ and $T$.

Suppose $p_{0}$ is another common fixed point of $f, g, S$ and T.Using (2) with $\alpha=1, x=p, y=p_{0}$ we can show that $p_{0}=p$. Thus $p$ is the unique common fixed point of $f, g, S$ and $T$.

Suppose $p_{1}$ is another common fixed point of $f$ and $S$. Using (2) with $\alpha=1$, $x=p_{1}, y=p$ we can show that $p_{1}=p$. Thus $p$ is the unique common fixed point of $f$ and $S$. Similarly we can show that $p$ is the unique common fixed point of $g$ and $T$.

Similarly the theorem holds when $g$ and $T$ are continuous.

Let $\mathbf{N}$ denote the set of all natural numbers. 
Corollary 2.2. Let $\left\{f_{i}\right\},\left\{g_{i}\right\},\left\{S_{i}\right\}$ and $\left\{T_{i}\right\}$ for every $i \in \mathbf{N}$ be selfmappings on $(X, M, *)$ such that

(1) there exist $i_{0}, j_{0}, m_{0}, n_{0} \in \mathbf{N}$ such that $f_{i_{0}}(X) \subseteq T_{n_{0}}(X)$ and

$$
g_{j_{0}}(X) \subseteq S_{m_{0}}(X)
$$

$M\left(f_{i} x, g_{j} y, t\right) \geq \phi\left(\begin{array}{l}M\left(S_{m} x, T_{n} y, t\right), M\left(f_{i} x, S_{m} x, t\right), M\left(g_{j} y, T_{n} y, t\right), \\ M\left(f_{i} x, T_{n} y, \alpha t\right), M\left(g_{j} y, S x,(2-\alpha) t\right)\end{array}\right)$

for every $x, y \in X$, for all $t>0$, for every $i, j, m, n \in \mathbf{N}$ and for every $\alpha \in(0,2)$, where $\phi \in \Phi$,

(3) the pairs $\left(f_{i_{0}}, S_{m_{0}}\right)$ and $\left(g_{j_{0}}, T_{n_{0}}\right)$ are weakly compatible,

(4) $f_{i_{0}}$ and $S_{m_{0}}$ are continuous or $g_{j_{0}}$ and $T_{n_{0}}$ are continuous,

(5) $S_{m} S_{m_{0}}=S_{m_{0}} S_{m}, S_{m} f_{i_{0}}=f_{i_{0}} S_{m}$ for all $m \in \mathbf{N}$ and $T_{n} T_{n_{0}}=T_{n_{0}} T_{n}$,

$T_{n} g_{j_{0}}=g_{j_{0}} T_{n}$ for all $n \in \mathbf{N}$.

Then the sequences of maps $\left\{f_{i}\right\},\left\{g_{j}\right\},\left\{S_{m}\right\}$ and $\left\{T_{n}\right\}$ have a unique common fixed point in $X$.

Proof. From Theorem 2.1 with $a=0, b=0$, the maps $f_{i_{0}}, g_{j_{0}}, S_{m_{0}}$, and $T_{n_{0}}$ have a unique common fixed point $p \in X$. Further $p$ is the unique common fixed point of $f_{i_{0}}$ and $S_{m_{0}}$ and $p$ is the unique common fixed point of $g_{j_{0}}$ and $T_{n_{0}}$. Suppose there exists $i \in \mathbf{N}$ such that $i \neq i_{0}$ with $f_{i}(p) \neq p$. Then from (2) with $j=j_{0}, m=m_{0}, n=n_{0}, \alpha=1$ and $x=y=p$ we have

$$
\begin{aligned}
M\left(f_{i} p, p, t\right)= & M\left(f_{i} p, g_{j_{0}} p, t\right) \\
& \geq \phi\left(\begin{array}{c}
M\left(S_{m_{0}} p, T_{n_{0}} p, t\right), M\left(f_{i} p, S_{m_{0}} p, t\right), M\left(g_{j_{0}} p, T_{n_{0}} p, t\right), \\
M\left(f_{i} p, T_{n_{0}} p, t\right), M\left(g_{j_{0}} p, S_{m_{0}} p, t\right)
\end{array}\right) \\
& =\phi\left(1, M\left(f_{i} p, p, t\right), 1, M\left(f_{i} p, p, t\right), 1\right) \\
& \geq \phi(r, r, r, r, r)>r
\end{aligned}
$$

where $r=M\left(f_{i} p, p, t\right)$. It is a contradiction.

Hence $f_{i}(p)=p$ for all $i \in \mathbf{N}$.

Similarly we can show that $g_{j}(p)=p$ for all $j \in \mathbf{N}$.

Now from $(5), S_{m}(p)=S_{m}\left(S_{m_{0}} p\right)=S_{m_{0}}\left(S_{m} p\right)$ and $S_{m}(p)=S_{m}\left(f_{i_{0}} p\right)=$ $f_{i_{0}}\left(S_{m} p\right)$. Thus $S_{m}(p)$ are common fixed points of $f_{i_{0}}$ and $S_{m_{0}}$ for each $m$. But $p$ is the unique common fixed point of $f_{i_{0}}$ and $S_{m_{0}}$. Therefore $S_{m}(p)=p$ for all $m$. Similarly we can show that $T_{n}(p)=p$ for all $n$. The uniqueness of $p$ follows from (2).

Corollary 2.3. Let $f_{1}, f_{2}, g_{1}, g_{2}$, Sand $T$ be self-mappings on $(X, M, *)$ such that

$$
\text { (1) } f_{1} f_{2}(X) \subseteq T(X) \text { and } g_{1} g_{2}(X) \subseteq S(X),
$$




$$
\begin{aligned}
& M\left(f_{1} f_{2} x, g_{1} g_{2} y, t\right) \\
& \geq \phi\left(\begin{array}{l}
M(S x, T y, t), M\left(f_{1} f_{2} x, S x, t\right), M\left(g_{1} g_{2} y, T y, t\right), \\
M\left(f_{1} f_{2} x, T y, \alpha t\right), M\left(g_{1} g_{2} y, S x,(2-\alpha) t\right)
\end{array}\right)
\end{aligned}
$$

for every $x, y \in X$, for all $t>0$ and for every $\alpha \in(0,2)$, where $\phi \in \Phi$,

(3) the pairs $\left(f_{1} f_{2}, S\right)$ and $\left(g_{1} g_{2}, T\right)$ are weakly compatible,

(4) $f_{1} f_{2}$ and $S$ are continuous or $g_{1} g_{2}$ and $T$ are continuous.

(5) $f_{1} f_{2}=f_{2} f_{1}, S f_{2}=f_{2} S, g_{1} g_{2}=g_{2} g_{1}, T g_{2}=g_{2} T$.

Then $f_{1}, f_{2}, g_{1}, g_{2}, S$ and $T$ have a unique common fixed point in $X$.

Proof. From Theorem 2.1 with $a=0, b=0$, the maps $f_{1} f_{2}, g_{1} g_{2}, S$ and $T$ have a unique common fixed point $p \in X$. Suppose $f_{2}(p) \neq p$.

From (2) with $x=f_{2} p, y=p, \alpha=1$ we have

$$
\begin{gathered}
M\left(f_{1} f_{2} f_{2} p, g_{1} g_{2} p, t\right) \\
\geq \phi\left(\begin{array}{l}
M\left(S f_{2} p, T p, t\right), M\left(f_{1} f_{2} f_{2} p, S f_{2} p, t\right), M\left(g_{1} g_{2} p, T p, t\right), \\
M\left(f_{1} f_{2} f_{2} p, T p, t\right), M\left(g_{1} g_{2} p, S f_{2} p, t\right)
\end{array}\right) \\
M\left(f_{2} p, p, t\right) \geq \phi\left(\begin{array}{l}
M\left(f_{2} p, p, t\right), M\left(f_{2} p, f_{2} p, t\right), M(p, p, t), \\
M\left(f_{2} p, p, t\right), M\left(p, f_{2} p, t\right)
\end{array}\right) \\
>M\left(f_{2} p, p, t\right)
\end{gathered}
$$

Hence $f_{2} p=p$. Now $f_{1} p=f_{1} f_{2} p=p$. Similarly we can show that $g_{1} p=$ $p=g_{2} p$. Uniqueness of $p$ follows from $(2)$.

\section{References}

[1] A.George and P. Veeramani, On some results in fuzzy metric space, Fuzzy Sets Systems, 64(1994), 395-399.

[2] I.Kramosil and J.Michalek , Fuzzy metric and statistical metric spaces, Kybernetica , 11 (1975), 326-334.

[3] J. Rodríguez López and S.Ramaguera , The Hausdorff fuzzy metric on compact sets, Fuzzy Sets Systems 147 (2004), 273-283.

[4] LA.Zadeh, Fuzzy sets, Inform and Control 8 (1965), 338-353.

[5] M.Grabiec, Fixed points in fuzzy metric spaces, Fuzzy Sets Systems 27 ( 1988), 385-389.

\section{Received: February 4, 2007}

